\title{
Analysis of Image Distortion on Magnetic Resonance Diffusion Weighted Imaging
}

\author{
Ah Rang Cho' ${ }^{1}$, Hae Kag Lee ${ }^{2}$, Heung Joon Yoo ${ }^{3}$, and Cheol-Soo Park ${ }^{4 *}$ \\ ${ }^{1}$ Department of Psychiatry, Kyung Hee University Hospital at Gangdong, School of Medicine, Kyung Hee University, \\ Seoul 05278, Korea \\ ${ }^{2}$ Department of Computer Science and Engineering, Soonchunhyang University, Asan 31538, Korea \\ ${ }^{3}$ Department of Radiology, Seoul Asan Medical Center, Seoul 05505, Korea \\ ${ }^{4}$ Department of Radiological Science, Hallym polytechnic university, Chuncheon 24210, Korea
}

(Received 1 October 2015, Received in final form 7 October 2015, Accepted 13 October 2015)

\begin{abstract}
The purpose of this study is to improve diagnostic efficiency of clinical study by setting up guidelines for more precise examination with a comparative analysis of signal intensity and image distortion depending on the location of $X$ axial of object when performing magnetic resonance diffusion weighted imaging (MR DWI) examination. We arranged the self-produced phantom with a $45 \mathrm{~mm}$ of interval from the core of 44 regent bottles that have a $16 \mathrm{~mm}$ of external diameter and $55 \mathrm{~mm}$ of height, and were placed in 4 rows and 11 columns in an acrylic box. We also filled up water and margarine to portrait the fat. We used 3T Skyra and 18 Channel Body array coil. We also obtained the coronal image with the direction of RL (right to left) by using scan slice thinkness $3 \mathrm{~mm}$, slice gap: $0 \mathrm{~mm}$, field of view (FOV): $450 \times 450 \mathrm{~mm}^{2}$, repetition time (TR): $5000 \mathrm{ms,} \mathrm{echo} \mathrm{time}$ (TE): 73/118 ms, Matrix: $126 \times 126$, slice number: 15, scan time: 9 min 45sec, number of excitations (NEX): 3 , phase encoding as a diffusion-weighted imaging parameter. In order to scan, we set b-value to $0 \mathrm{~s} / \mathrm{mm}^{2}, 400 \mathrm{~s} /$ $\mathrm{mm}^{2}$, and $1,400 \mathrm{~s} / \mathrm{mm}^{2}$, and obtained $\mathrm{T} 2$ fat saturation image. Then we did a comparative analysis on the differences between image distortion and signal intensity depending on the location of $X$ axial based on isocenter of patient's table. We used "Image $J$ " as a comparative analysis programme, and used SPSS v18.0 as a statistic programme. There was not much difference between image distortion and signal intensity on fat and water from $T 2$ fat saturation image. But, the average value depends on the location of $X$ axial was statistically significant $(p<0.05)$. From DWI image, when b-value was 0 and 400 , there was no significant difference up to $2^{\text {nd }}$ columns right to left from the core of patient's table, however, there was a decline in signal intensity and image distortion from the $3^{\text {rd }}$ columns and they started to decrease rapidly at the $4^{\text {th }}$ columns. When b-value was 1,400 , there was not much difference between the $1^{\text {st }}$ row right to left from the core of patient's table, however, image distortion started to appear from the $2^{\text {nd }}$ columns with no change in signal intensity, the signal was getting decreased from the $3^{\text {rd }}$ columns, and both signal intensity and image distortion started to get decreased rapidly. At this moment, the reagent bottles from outside out of 11 reagent bottles were not verified from the image, and only 9 reagent bottles were verified. However, it was not possible to verify anything from the $5^{\text {th }}$ columns. But, the average value depends on the location of $X$ axial was statistically significant. On T2 FS image, there was a significant decline in image distortion and signal intensity over $180 \mathrm{~mm}$ from the core of patient's table. On diffusion-weighted image, there was a significant decline in image distortion and signal intensity over $90 \mathrm{~mm}$, and they became unverifiable over $180 \mathrm{~mm}$. Therefore, we should make an image that has a diagnostic value from examinations that are hard to locate patient's position.
\end{abstract}

Keywords : T2-Fat Saturation, magnetic resonance diffusion-Weighted Image, b-value, iso-center

\section{Introduction}

Magnetic resonance imaging (MRI) is widely used for diagnosis in real clinical cases with the advent of simple

CThe Korean Magnetics Society. All rights reserved.

*Corresponding author: Tel: $+82-33-240-9350$

Fax: +82-33-240-9350, e-mail: pcs3109@hsc.ac.kr weighted imaging method that has contrast effect, highspeed imaging, magnetic resonance spectroscopy, magnetic resonance angiography, brain functional imaging, diffusion imaging as well as perfusion imaging. Of all, diffusion weighted magnetic resonance imaging (MRI DWI) is not only being successfully used for distinguishing the ingredients of central nervous system, acute stroke diagnosis, and brain tumor, but also is applied to 
leukoencephalopathy (multiple sclerosis, wallerian degeneration, and delayed myelination), diffuse axonal injury, schizophrenia, and cognitive impairment. Recently, it is used for diagnosing brain diseases, epigastric area such as hepatobiliary and pancreatic disease, hypogastric area such as prostate and gynecological diseases, and colorectal cancer. Also, it is used for distinguishing the features of lump and musculoskeletal system tumor that occurred in women's breast [1-6]. According to the first diffusion gradient magnetic field, there will be a phase difference between moving and non-moving quantum, and then, if the second gradient magnetic field got caught, all of non-moving quantum will be refocused while moving quantum is being less refocused because of the continuos diffusion motion causing the decline in signal intensity. The degree of DRI DWI's signal intensity will be decreased when diffusion coefficient $\left(\mathrm{mm}^{2} / \mathrm{sec}\right)$ or gradient factor is big. That is to say, the difference between water molecule's diffusion motion and water molecule's diffusion existed in an abnormal cell is expressed as the image contrast. When it comes to the cerebral infection, it is expressed with a bright signal because there has been a decline in diffusion motion of cells and water molecules due to vasogenic edema occurred by cardiovascular disease. In case of malignant cancer, it is brightly portrayed because of the decline of diffusion motion of water molecules between tumor cells due to the undifferentiated cellulation. That is to say, diffusion is a very innovative image diagnosing method that is able to detect the tissue's diffusion degree within an organism [7, 8]. In addition, By using frequency encoding gradient appeared by EPI (echo planar imaging), we can obtain kspace data that is capable of composing a sliced image during the $\mathrm{T} 2{ }^{*}$ dephase that was occurred by excitation
RF pulse. This method is the fastest way to compose an image out of MRI techniques with a short scans time and no artifact. However, unlike other techniques, this technique obtains the data during a single TR after approving 90 and 180 degree pulse, therefore it is more sensitive to magnetic susceptibility because it uses gradient coil but the re-focus pulse after approving 180 degree pulse. For this reason, there are image distortion and signal intensity decline, especially when examining the parts that are away from magnetic field's Iso-center such as shoulders, elbow, and wrist $[9,10]$. Therefore, this study self produced the phantom in order to find out the difference in image distortion and signal intensity depending on the location of $\mathrm{X}$ axial within the magnetic field when performing MRI DWI examination. Also, the study was to obtain the image by differentiating $b$-value and doing a comparative analysis on the obtained image in order to set guidelines for more precise examination and improve the diagnostic efficiency in clinical cases.

\section{Subject and Method}

\subsection{Phantom's production}

In order to measure the signal intensity and image distortion depending on the location of $\mathrm{X}$ axial within the magnetic field, we produced the phantom so that we could fully cover X axial of patient's table, and arranged the phantoms with a $45 \mathrm{~mm}$ of interval from the core of 44 round regent bottles that were placed in 4 rows and 11 columns on a $490 \mathrm{~mm} \times 150 \mathrm{~mm}$ of acrylic plate; the regent bottle that we used has a $16 \mathrm{~mm}$ of external diameter and $55 \mathrm{~mm}$ of height. We filled up the $1^{\text {st }}$ and $3^{\text {rd }}$ rows with water in order to portrait the fat, and filled up the $2^{\text {nd }}$ and $4^{\text {th }}$ with distilled water. We concealed every
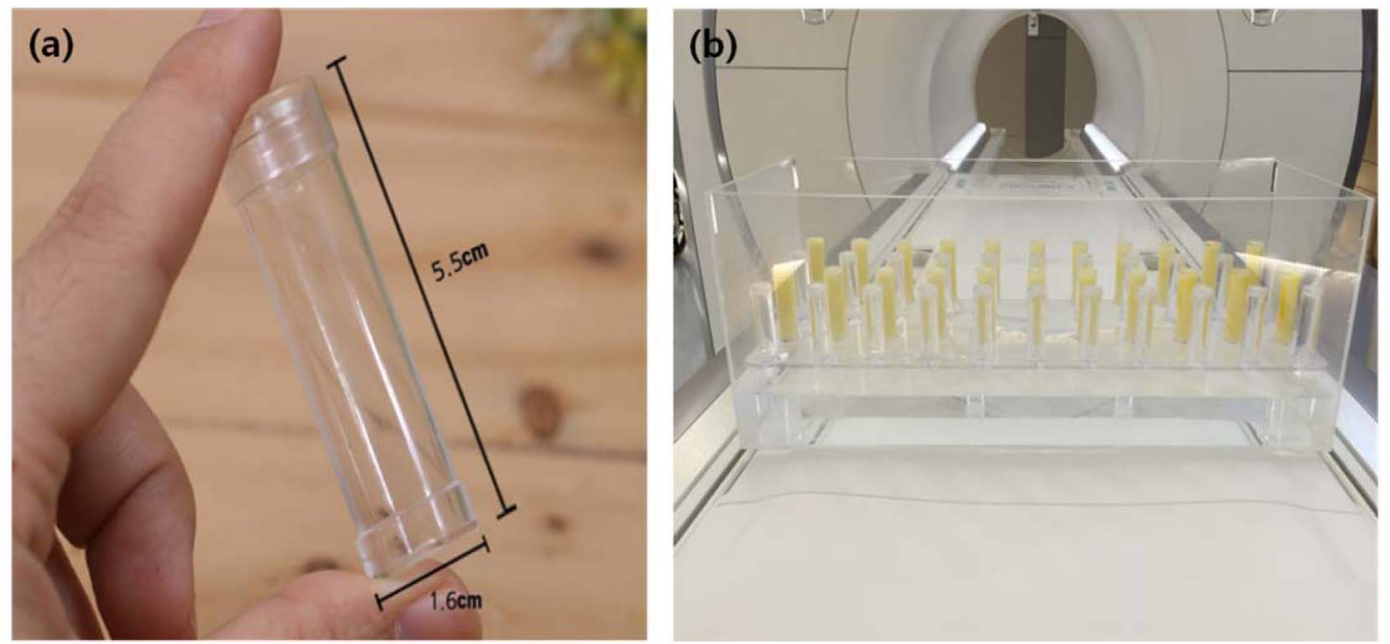

Fig. 1. (Color online) A reagent bottle (A) and self-produced phantom (B). 
regent bottle to prevent them from any foreign body and air. Then we put the acrylic plate $60 \mathrm{~mm}$ away from 500 $\mathrm{mm} \times 170 \mathrm{~mm} \times 170 \mathrm{~mm}$ of acrylic box's bottom. We filled up the $60 \mathrm{~mm}$ of space between acrylic box's bottom and acrylic plate with distilled water to increase the magnetic susceptibility (Fig. 1).

\section{2. tools and scan parameter}

We used 3T Skyra (Siemens Germany) and 18Channel Body array coil. We also obtained the coronal image with the direction of RL (right to left) by using scan slice thickness $3 \mathrm{~mm}$, slice gap: $0 \mathrm{~mm}$, field of view (FOV): $450 \times 450$, repetition time (TR): $5000 \mathrm{~ms}$, echo time (TE): $73 / 118 \mathrm{~ms}$, Matrix: $126 \times 126$, slice number: 15 , scan time: $9 \mathrm{~min} 45 \mathrm{sec}$, number of excitations (NEX): 3, phase encoding as a diffusion-weighted imaging parameter. In order to scan, we set b-value to $0 \mathrm{~s} / \mathrm{mm}^{2}, 400 \mathrm{~s} /$ $\mathrm{mm}^{2}$, and $1,400 \mathrm{~s} / \mathrm{mm}^{2}$, and obtained $\mathrm{T} 2$ fat saturation image. For parameter, we used scan slice thickness $3 \mathrm{~mm}$, slice gap: $0 \mathrm{~mm}$, FOV: $450 \times 450$, TR: 3200, TE: 86 , Matrix: $320 \times 320$, slice number: 15 , scan time: $2 \min 43$ sec, NEX: 2, (Short-scar Periareolar-Inferior pedicle Reduction) SPAIR technique.

\subsection{Study Method}

In order to find out if image distortion and signal intensity got worse when the regent bottle was away from the core, we always placed the self-produced phantom in the middle of patient table. First, we were able to obtain T2 fat saturation (FS) image using spin echo (SE) technique to compare with DWI test using Echo-planar imaging (EPI). Second, we obtained the image through 10 times by setting b-value as 0,400 , and 1,00 in order to find out how b-value's size impact on an image distortion when doing DWI examination. A gradient pulse encodes the spins with a characteristic phase dependent on spatial position along the gradient direction, as depicted in Fig. 2. The equation that describes the amount of phase, $\varphi$, imparted on each spin after a gradient pulse is applied, on the $\mathrm{x}$-axis for example, is given by [9]

$$
\varphi=\gamma \int_{0}^{t} G\left(t^{\prime}\right) \times x\left(t^{\prime}\right) d t^{\prime} .
$$

A second gradient of equal time and equal and opposite amplitude will remove the phase that was set up by the first gradient. For static spins, where no net translational movement has taken place, the phase of all the spins will be brought back together, as depicted in Fig. 2. If diffusion has occurred then some intravoxel phase dispersion will remain. Assuming movement only occurs between the gradients, with amplitude GD and duration $\delta$, the change in phase, $\Delta \varphi$, for a spin that has moved an amount $\Delta x$ can be calculated from Eq. (2) to be [9]

$$
\Delta \varphi=\gamma \delta G_{D} \Delta x=q \Delta x .
$$

The result is an attenuation of the signal amplitude that is due to the destructive interference of the spins with varying phase. The following equation describes a general signal attenuation due to a distribution of phase [9] :

$$
S=S_{0} \int e^{i \Delta \varphi} P(\Delta x) d \Delta x,
$$

where $P(\Delta x)$ is the probability distribution of the ensemble of diffusing spins. The Einstein relation states that for free diffusion, $P(\Delta x)$ is a Gaussian $[9,10]$. Substituting in Eq. (2) for $\Delta \varphi$ and assuming free diffusion,

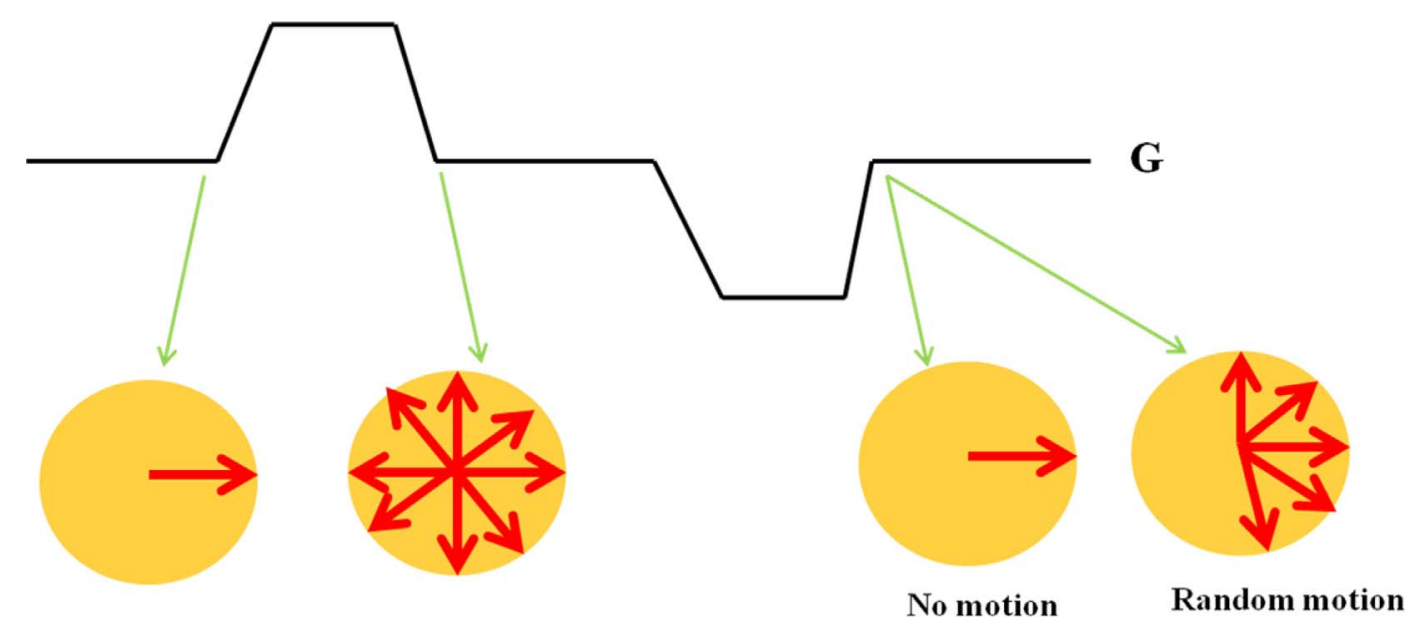

Fig. 2. (Color online) Before a diffusion gradient is applied, the spins within a voxel have identical phase. The first diffusion gradient encodes each spin with a phase dependant on its position. If no motion has occurred, the second diffusion gradient, that has the same strength and opposite polarity, will rewind the phase of each spin. 
Eq. (3) is written as [9]

$$
S=\frac{S_{0}}{\sqrt{2 \pi \sigma_{\Delta x}^{2}}} \int e^{i q \Delta x} e^{-\frac{(\Delta x)^{2}}{2 \sigma_{\Delta x}^{2}}} d \Delta x .
$$

By way of the Fourier Transform, this integral solves to [9]

$$
S=S_{0} e^{-\frac{q^{2}}{2} \sigma_{\Delta x}^{2}} \text {. }
$$

$\sigma_{\Delta x}^{2}$ is the variance of the Gaussian probability function, and for free diffusion has the form [9]

$$
\begin{aligned}
& \sigma_{\Delta x}^{2}=\left[\Delta x^{2}\right]=2 D T_{\text {diff }}, \\
& S=S_{0} e^{-\left(\gamma \delta G_{D}\right)^{2 D T_{d i f f}}} .
\end{aligned}
$$

The variance of the Gaussian is a function of both the diffusion coefficient, $D$, and the total amount of time spins are allowed to diffuse, Tdiff. Substituting Eq. (6) into Eq. (5) and writing out $q$ in its full form, the equation for signal attenuation due to diffusion is given by [9]

\subsection{Image Distortion Measurement}

We measured the image distortion with the external diameter of self-produced phantom's round regent bottle. On the assumption that image distortion was minimum in the core of magnetic field, we did a comparative analysis on the different size of T2 FS image and DWI image of regent bottles that are $45 \mathrm{~mm}$ away from one another right to left as an image distortion, and computed the average value of the $1^{\text {st }}$ and $3^{\text {rd }}$ row that are filled with margarine and $2^{\text {nd }}$ and $4^{\text {th }}$ row that are filled with water depending on the location of $\mathrm{X}$ axial. We made sure to regulate the coronal image to non-artifact image on T2 FS image and used Image J programme to measure the image area. In order to find out the statistical significance, we used SPSS v18.0 statistic programme and conducted Duncan post-analysis in order to find out the significance between each group depending on the location of $\mathrm{X}$ axial.

\subsection{Signal Intensity Measurement}

We used "Image J" as a comparative analysis programme, and used SPSS (SPSS v18.0, USA, Chicago) as a statistic programme. Just like the image distortion measurement, we did a comparative analysis on signal intensity (SI) using Image J programme from T2 FS image and DWI image of regent bottles that are arranged with a $45 \mathrm{~mm}$ of interval right to left based on the regent bottle in the middle of patient table. In order to find out the statistical significance, we did some statistics as well.

\section{Results}

From the T2 FS image using SE technique, there was not much difference of area from $\mathrm{X}_{-3}$ to $\mathrm{X}_{3}$ based on the core of patient table, $\mathrm{X}_{0}$. However, as the regent bottles grew away from the core just like the location of $\mathrm{X}_{-4}, \mathrm{X}_{4}$, we could find the changes of the area. Signal intensity seemed to be normal from $X_{-3}$ to $X_{3}$, but it showed a decline from the location of $\mathrm{X}_{-4}$ and $\mathrm{X}_{3}$. From DWI image, when b-value was 0 or 400 , there was not much difference from $\mathrm{X}_{-2}$ to $\mathrm{X}_{2}$, but we found the change of the
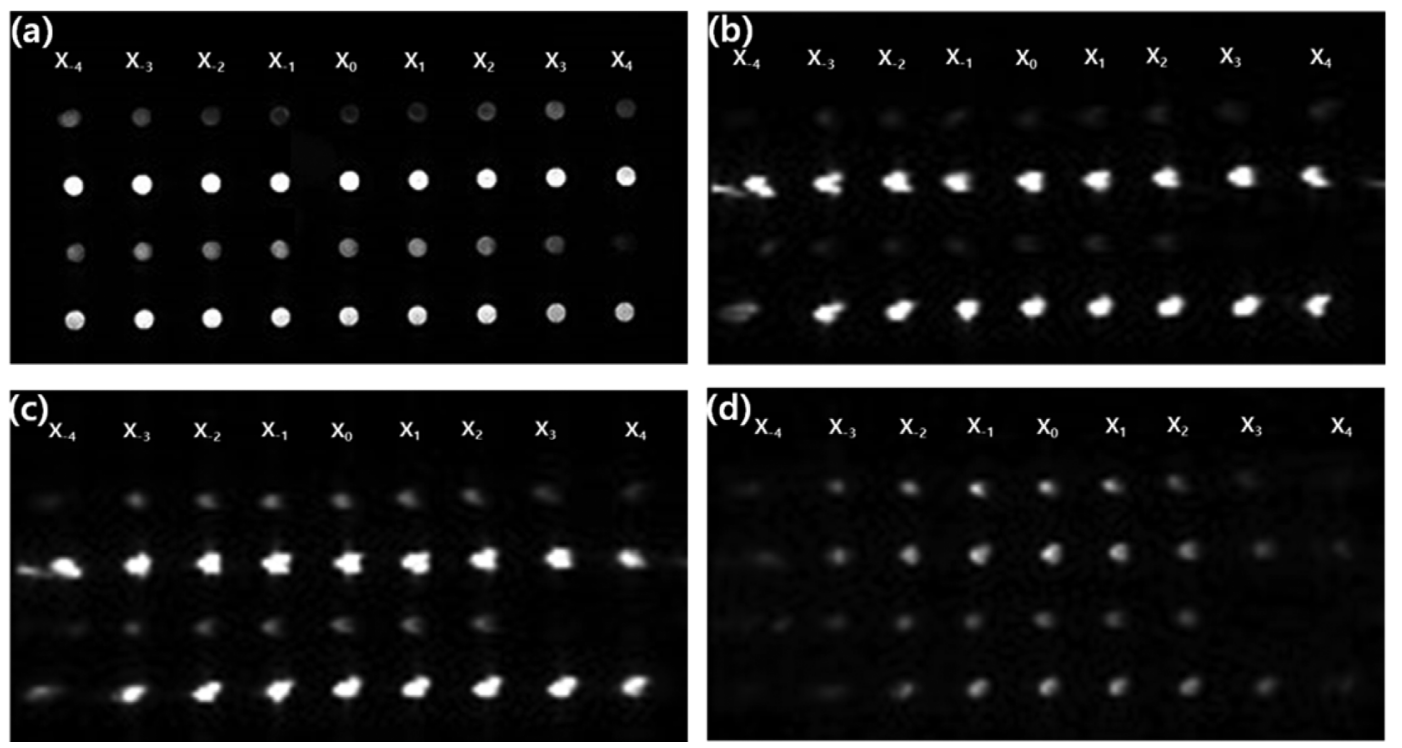

Fig. 3. (a) T2 FS image, (b) DWI b-vale $0 \mathrm{~s} / \mathrm{mm}^{2}$ image (c) DWI b-vale $400 \mathrm{~s} / \mathrm{mm}^{2}$ image (d) DWI b-vale $1400 \mathrm{~s} / \mathrm{mm}^{2}$ image. 
Table 1. Changes of Area depending on the location of $X$ axial of patient table from MR image.

\begin{tabular}{cccccccccccc}
\hline \hline Image & Division & $\mathrm{X}_{-4}$ & $\mathrm{X}_{-3}$ & $\mathrm{X}_{-2}$ & $\mathrm{X}_{-1}$ & $\mathrm{X}_{0}$ & $\mathrm{X}_{1}$ & $\mathrm{X}_{2}$ & $\mathrm{X}_{3}$ & $\mathrm{X}_{4}$ & $\mathrm{P}$ \\
\hline \multirow{2}{*}{ T2 FS } & fat & 126.56 & 136.21 & 136.38 & 136.45 & 136.45 & 135.84 & 135.21 & 136.09 & 128.43 & 0 \\
& water & 148.25 & 157.18 & 157.81 & 158.2 & 158.2 & 158.02 & 157.76 & 157.45 & 152.21 & 0 \\
\hline \multirow{2}{*}{ DWI b-value 0 image } & fat & 126.56 & 136.21 & 136.38 & 136.45 & 136.45 & 135.84 & 135.21 & 136.09 & 128.43 & 0 \\
& water & 148.25 & 157.18 & 157.81 & 158.2 & 158.2 & 158.02 & 157.76 & 157.45 & 152.21 & 0 \\
\hline \multirow{2}{*}{ DWI b-value 400 image } & fat & 82.35 & 109.42 & 149.23 & 150.98 & 153.06 & 153.04 & 150.18 & 108.2 & 65.18 & 0 \\
& water & 202.04 & 267.86 & 302.24 & 328.29 & 331.63 & 329.5 & 262.3 & 242.25 & 153.06 & 0 \\
\hline \multirow{2}{*}{ DWI b-value 1400 image } & fat & 51.02 & 102.04 & 114.24 & 150.06 & 153.24 & 153.04 & 101.52 & 99.98 & 76.53 & 0 \\
& water & 76.53 & 127.55 & 138.68 & 140.31 & 153.08 & 142.56 & 140.24 & 114.8 & 74.52 & 0 \\
\hline
\end{tabular}

FS: fat saturation, DWI: diffusion weighted image

area and decline of signal intensity from $\mathrm{X}_{-3}$ and $\mathrm{X}_{3}$. Besides, there was a rapid change of area and decline of signal intensity at $\mathrm{X}_{-4}$ and $\mathrm{X}_{4}$. When b-value was 1,400 , there was not much difference of the area from $X_{-1}$ to $X_{1}$, but we found the change of the area from $\mathrm{X}_{-2}$ and $\mathrm{X}_{2}$. Signal intensity seemed normal from $\mathrm{X}_{-2}$ to $\mathrm{X}_{2}$, but we could find a decline from $\mathrm{X}_{-3}, \mathrm{X}_{3}$, and signal intensity and the area started to become more rapid at $\mathrm{X}_{-4}$ and $\mathrm{X}_{4}$. At this moment, we were not able to verify the very outside of regent bottle out of 11 regent bottles, and we could only verify the 9 regent bottles from the image (Fig. 3). From T2 Fs image, fat's $\mathrm{X}_{0}$ Area was 136.45, the standard deviation from $X_{-3}$ to $X_{3}$ was $136.09 \pm 0.45$, and the standard deviation from $\mathrm{X}_{-4}$ to $\mathrm{X}_{4}$ was $134.18 \pm 3.84$. On the other hand, water's $X_{0}$ Area was 158.20 , standard deviation from $X_{-3}$ to $X_{3}$ was $157.80 \pm 0.38$, and the standard deviation from $\mathrm{X}_{-4}$ to $\mathrm{X}_{4}$ was $156.12 \pm 3.50$. The average value depending on the location of $\mathrm{X}$ axial was significant $(p<0.05)$ (Table 1). From DWI b-value 0, fat's standard deviation from $\mathrm{X}_{-2}$ to $\mathrm{X}_{2}$ was $153.00 \pm 0.11$, from $\mathrm{X}_{-3}$ to $\mathrm{X}_{3}$ was $140.83 \pm 20.80$, and from $\mathrm{X}_{-4}$ to $\mathrm{X}_{4}$ was $125.62 \pm 35.22$. Water's standard deviation from $\mathrm{X}_{-2}$ to $\mathrm{X}_{2}$ was $264.98 \pm 4.02$, from $\mathrm{X}_{-3}$ to $\mathrm{X}_{3}$ was $255.50 \pm 23.52$, and from $\mathrm{X}_{-4}$ to $\mathrm{X}_{4}$ was $238.40 \pm 41.47$. The average value depending on the location of $\mathrm{X}$ axial was significant $(p<0.05)$ (Table 1). From DWI b-value 400, fat's standard deviation from to $\mathrm{X}_{-2}$ to $\mathrm{X}_{2}$ was $151.30 \pm 2.04$, from $\mathrm{X}_{-3}$ to $X_{3}$ was $139.16 \pm 20.70$, and from $X_{-4}$ to $X_{4}$ was 124.63 \pm 34.14 . Water's standard deviation from $\mathrm{X}_{-2}$ to $\mathrm{X}_{2}$ was $310.80 \pm 29.64$, from $X_{-3}$ to $X_{3}$ was $294.87 \pm 37.15$, and from $\mathrm{X}_{-4}$ to $\mathrm{X}_{4}$ was $268.78 \pm 62.14$. The average value depending on the location of $\mathrm{X}$ axial was significant $(p<$ 0.05 ) (Table 1). From DWI b-value 1,400, fat's standard deviation from $\mathrm{X}_{-1}$ to $\mathrm{X}_{1}$ was $152.11 \pm 1.78$, from $\mathrm{X}_{-3}$ to $\mathrm{X}_{3}$ was $124.87 \pm 25.92$, and from $\mathrm{X}_{-4}$ to $\mathrm{X}_{4}$ was $111.30 \pm$ 35.64. Water's standard deviation from $\mathrm{X}_{-1}$ to $\mathrm{X}_{1}$ was $145.32 \pm 6.82$, from $X_{-3}$ to $X_{3}$ was $142.97 \pm 12.22$, and from $\mathrm{X}_{-4}$ to $\mathrm{X}_{4}$ was $123.14 \pm 28.99$. The average value depending on the location of $\mathrm{X}$ axial was significant $(p<0.05)$ (Table 1).

\section{Discussion}

Through this study, we found that the location of $\mathrm{X}$ axial of magnet field has an impact on MRI DWI examination. Therefore, now that we know we should near to the core of patient table as much as possible when performing MRI DWI examination that is used for diagnosing the tumor from the limb, musculoskeletal bone, and soft tissue in order to get clinically useful images. DWI examination has been widely used in a real clinic with the various studies. However, since this examination is very sensitive to the movement, it was hard to verify the motion artifact and apply it without the reinforcement of $\mathrm{H} / \mathrm{W}$ and $\mathrm{S} / \mathrm{W}$ [11-14]. Of all, with the development of EPI, the possibility of clinical application has increased because EPI is the fastest out of MRI methods that requires the extra hardware unlike other methods. In addition, it is transformed from Spin-Echo and Gradient-Echo sequence. Spin-Echo image approves 180-degree pulse, minimizes the inhomogeneity of the main magnetic field, and obtains $\mathrm{T} 1$ and $\mathrm{T} 2$ strengthened image. On the other hand, EPI image transformed by Gradient-Echo requires much faster and bigger gradient pulse than the general system. It also requires the higher speed of digital-to-analog converter. However, even though we use the right hardware, it is undeniable that it has a lower image resolution and SNR compared to other imaging methods $[15,16]$. Since DWI technique came from the phenomena that water molecule moves from high molecular density to low molecular density, it require a strong gradient magnetic field, so the inhomogeneity within the main magnetic field gets increased. However, the DWI technique that we used in this study was 
different. It was not the single-shot EPI DWI technique that filled up K-space without segmenting readout direction but RESOLVE DWI technique that was provided from Skyra from Siemens. This new technique is called diffusion weighted EPI that segments K-space to the readout direction and fills up K-space with a multi-shot method. Therefore, we are able to get a clear image with a higher spatial resolution with a lower susceptibility artifact and T2-star bluming by improving the sampling speed and decreasing TE [17].

Nevertheless, there will be an image distortion and signal intensity decline when growing apart from the core of the patient's table because the location of $\mathrm{X}$ axial within the magnetic field has a huge impact. As the limitations of this study, even though the 3.0 Tesla equipment that we used for this study was capable of improving SNR compared to 1.5 Tesla's low magnetic field MRI equipment, we did not compare the differences depending on the magnetic intensity based on the theory that homogeneity will get decreased when there is a higher magnetic field. Also, we limited the ingredients inside of the regent bottle of phantom to only two kinds. Therefore, we should use the phantom that has more various ingredients and assess the clinical usability in the future. MRI DWI examination is widely used for diagnosing the central nervous system as well as distinguishing the features of musculoskeletal and abdominal tumor, and is providing great diagnostic information.

For these reasons, we come to the conclusion through the study in order to improve the diagnostic efficiency of DWI examination. Considering the fact that there is a 45 $\mathrm{mm}$ of interval between each regent bottle, there was a significant difference when growing more than $180 \mathrm{~mm}$ away from the core of patient's table on T2 FS image. However, on diffusion-weighted image, there was a significant decline in image distortion and signal intensity over $90 \mathrm{~mm}$, and they became unverifiable over $180 \mathrm{~mm}$. In addition, the more $b$-value increases, the more impact occurs depending on the location of $\mathrm{X}$ axial. Therefore, we should make a valuable image in diagnosis with being more careful in an examination that has a difficulty of locating patient's position.

\section{Conclusions}

From the T2 FS image, there were significant image distortion and signal intensity's decline $180 \mathrm{~mm}$ away from the core of patient table. From DWI image, there were significant image distortion and signal intensity's decline $90 \mathrm{~mm}$ away from the core, and they became unverifiable over $180 \mathrm{~mm}$ away from the core. Therefore, we should make a valuable image in diagnosis with being more careful in an examination that has a difficulty of locating patient's position.

\section{Acknowledgements}

Ah Rang Cho and Hae Kag Lee equally contributed to this work. They are co-first authors. This work was supported in part by the Soonchunhyang university Research Fund.

\section{References}

[1] D. Le Bihan, E. Breton, D. Lallemand, M. L. Aubin, J. Vignaud, and M. LavalJeantet, Radiology. 168, 497 (1988).

[2] R. G. Gonzalez, P. W. Schaefer, F. S. Buonanno, L. H. Schwamm, R. F. Budzik, G. Rordorf, B. Wang, A. G. Sorensen, and W. J. Koroshetz, Radiology. 210, 155 (1999).

[3] M. P. Marks, A. de Crespigny, D. Lentz, D. R. Enzmann, G. W. Albers, and M. E. Moseley, Radiology. 199, 403 (1996).

[4] R. D. Tien, G. J. Felsberg, H. Friedman, M. Brown, and J. MacFall, Am. J. Roentgenol. 162, 671 (1994).

[5] J. S. Tsuruda, W. M. Chew, M. E. Moseley, and D. Norman, Am. J. Roentgenol. 155, 1059 (1990).

[6] C. F. Maier, Y. Paran, P. Bendel, B. K. Rutt, and H. Degani, Magn. Reson. Med. 37, 576 (1997).

[7] J. H. Cho, H. K. Lee, K. R. Dong, W. K. Chung, S. C. Kim, and T. J. Ji, Appl. Magn. Reson. 42, 531 (2012).

[8] T. Neumann-Haefelin, H. J. Wittsack, F. Wenserski, M. Siebler, R. J. Seitz, U. Mödder, and H. J. Freund, Stroke. 30, 1591 (1999).

[9] J. E. Sarlls, The University of Arizona 146, 3215376 (2006).

[10] A. Gholipour, N. Kehtarnavaz, B. Scherrer, and S. K. Warfield, Conf. Proc. IEEE. Eng. Med. Biol. Soc. 2011, 6997 (2011).

[11] A. W. Anderson and J. C. Gore, Magn. Reson. Med. 32, 379 (1994).

[12] P. J. Basser, J. Mattiello, and D. LeBihan, J. Magn. Reson. 103, 247 (1994).

[13] C. Beaulieu, NMR Biomed. 15, 435 (2002).

[14] K. Butts, J. Pauly, A. de Crespigny, and M. Moseley, Magn. Reson. Med. 38, 741 (1997).

[15] P. Mukherjee, S. W. Chung, J. I. Berman, C. P. Hess, and R. G. Henry, American Journal of Neuroradiology. 29, 843 (2008).

[16] O. Josephs, R. Deichmann, and R. Turner, NeuroImage. 11, S543 (2000).

[17] D. A. Porter, and R. M. Heidemann, Magn. Reson. Med. 62, 468 (2009). 